Methods Women attending health services across Australia provided a self-collected (vaginal) or clinician-collected (cervical) specimen for HPV genotyping (Roche Linear Array) and completed a questionnaire. HPV vaccination status was validated against the National HPV Vaccination Program Register. Odds ratios (ORs) and 95\% confidence intervals (CI) were calculated for factors associated with detection of any oncogenic HPV (HPV16/18/31/33/35/39/45/51/52/56/58/59/66/68).

Results Among 1,643 women, vaccine coverage ( $\geq$ one dose) was $61.5 \%(69.1 \%, 58.7 \%$ and $41.1 \%$ among those $18-24$, 25-29 and 30+ years, respectively). Oncogenic HPV prevalence was $25.4 \%$ (95\% CI: 23.2-27.6\%). In univariable analysis, risk factors for detection included younger age (ptrend $<0.001)$, being a current smoker $(p=0.05)$, and reporting more lifetime ( $\mathrm{p}$-trend $<0.001$ ) and recent (last 12 months) sexual partners (p-trend $<0.001$ ). In multivariable analysis, younger age (adjusted $\mathrm{OR}=1.33$ [1.17-1.52]), more lifetime (adjusted $\mathrm{OR}=1.33[1.16-1.52]$ ) and recent sexual partners (adjusted $\mathrm{OR}=2.37$ [1.77-3.16]) remained significant. There were no associations with socioeconomic status, area of residence or vaccination history. HPV16/18 prevalence was $1.4 \%$ (0.9-2.1\%). In univariable analysis, risk factors for detection included older age $(p=0.05)$, being non-Australian born $(p=0.05)$, and being unvaccinated $(p<0.001)$. In multivariable analysis, being unvaccinated remained the only factor significantly associated with HPV16/18 detection (adjusted OR=8.61 [2.45-30.25]). There were no associations with area of residence, socioeconomic status, or sexual behaviour.

Conclusion Oncogenic HPV was commonly detected among young Australian women; prevalence was influenced by risk factors related to sexual behaviour. In contrast, prevalence of quadrivalent vaccine-targeted types $16 / 18$ was very low and influenced only by vaccination status. Vaccination has changed the epidemiology of HPV infection in Australia.

Disclosure No significant relationships.

\section{P823 COMPARISON OF COPAN URISPONGETM TO COLLI-PEE FOR THE COLLECTION OF URINE FOR HPV DETECTION WITH MOLECULAR ASSAYS}

${ }^{1}$ Santina Castriciano*, ${ }^{2}$ Marianna Martinelli. 'Copan Italia Spa, Scientific Affairs, Hamilton Canada; ${ }^{2}$ Department of Medicine and Surgery, University of Milano-Bicocca, Monza, Italy

\subsection{6/sextrans-2019-sti.868}

Background Urine specimen collection is non-invasive and better accepted by patients for HPV and STI screening. The Copan UriSponge ${ }^{\mathrm{TM}}$, a Liquid Based Microbiology device, used for urine collection for culture and molecular assays. It consists of a leak-proof tube with a screw cap containing a plastic stick with sponges attached that absorb and retain the urine sample during transport preventing bacterial overgrowth. The objective of this study was to compare the UriSponge ${ }^{\mathrm{TM}}$ (US) to the Colli-Pee (CP) (Novosanis) urine devices for selfcollection of first void urine (FVU) for the detection of HPV with the Seegene AnyplexII ${ }^{\mathrm{TM}}$-HPV28-assay.

Methods FVU, from 72 patients with a recent diagnosis of cervical dysplasia, attending the Gynecology clinic for colposcopy. $20 \mathrm{ml}$ of first-void urine were collected using Colli$\mathrm{Pee}^{\mathrm{TM}}$ and an additional aliquot of urine was collected in sterile containers to saturate UriSponge ${ }^{\mathrm{TM}}$. Nucleic acids were extracted by NucliSENS easyMAG (bioMérieux) from US and $\mathrm{CP}$ urine. HPV detection was performed using AnyplexII ${ }^{\mathrm{TM}}$
HPV28-Assay. Sample cellularity was evaluated with a quantitative real-time PCR detecting human CCR5 gene.

Results Out of 72 urines tested 24 (33.3\%) were positive for HR-HPV, $16(22.2 \%)$ for HR+LR, 8 (11.1\%) for LR and 24 $(22.2 \%)$ were negative from both US and CP. Comparable cellularity was present in US and $\mathrm{CP}$ with a mean value of 2.09E +06. Optimal concordance for all HR- HPV genotypes compared to cervical sample was demonstrated for US and CP. HPV 16, 18, 51 and 31 were most frequently HR-HPV genotypes.

Conclusion Data obtained in this study demonstrated that the Copan UriSponge ${ }^{\mathrm{TM}}$ detected all HR-HPV positive samples and good cellularity compared to the cervical swab when compared to the Novosanis Colli-Pee ${ }^{\mathrm{TM}}$ using the Seegene Anyplex $^{\mathrm{TM}}$ II HPV28 assay. The UriSponge ${ }^{\mathrm{TM}}$ is easy to use for urine self-collection, it's not bulky, can be conveniently shipped by mail at a relatively low cost.

Disclosure No significant relationships.

\section{P824 ACCURACY OF CERVICAL CANCER SCREENING USING A SELF-COLLECTED VIAL FOR HPV DNA TESTING AMONG ADULT WOMEN IN SUB-SAHARAN AFRICA} ${ }^{3}$ Hélène Péré, ${ }^{3}$ David Veyer, ${ }^{4}$ Chatté Adawaye, ${ }^{3}$ Mathieu Matta, ${ }^{3}$ Leman Robin, ${ }^{5}$ Serge Tonen-Wolyec, ${ }^{6}$ Moll Mahamat Tcheguena, ${ }^{7}$ Ali Mahamat Moussa, ${ }^{8}$ Donato Koyalta, ${ }^{3}$ Laurent Bélec. 'Service de Gynécologie-Obstétrique, Hôpital de la Mère et de l'Enfant, and Cabinet Médical de Gynécologie Obstétrique 'La Renaissance Plus', N'Djamena, Chad; ${ }^{2}$ Ecole Doctorale Régionale d'Infectiologie Tropicale de Franceville, Franceville, Gabon; ${ }^{3}$ Laboratoire de Virologie, Hôpital Européen Georges Pompidou and Faculté de Médecine Paris Descartes, Université Paris Descartes (Paris V), Sorbonne Paris Cité, Paris, France; ${ }^{4}$ Institut National Supérieur des Sciences et Techniques d'Abéché, N'Djamena, Chad; ${ }^{5}$ Faculté de Médecine et de Pharmacie, Université de Kisangani, Kisangani, Republic of Congo; ${ }^{6}$ Faculté de Médecine, N'Djamena, Chad; ${ }^{7}$ Service de Gastro-entérologie, Hôpital Général de Référence Nationale, N'Djamena, Chad; ${ }^{8}$ UNAIDS, N'Djamena, Chad

10.1136/sextrans-2019-sti.869

Background Cervical cancer is caused by HR-HPV infection. Self-collection of genital specimens and HPV DNA testing are methods increasing screening rates. The GYNAUTO-CHAD study compared the acceptability and HPV DNA diagnostic accuracy of clinician-collected endocervical sample with swab (as reference collection) and genital self-collection method with veil (V-Veil-Up Gyn Collection Device, V-Veil-Up Pharma Ltd., Nicosia, Cyprus) in adult women living in N'Djamena, Chad.

Methods Five of the 10 districts of N'Djamena were randomly selected for inclusion. Peer educators contacted women to participate to the survey by coming to the clinic for women's sexual health 'La Renaissance Plus'. A clinician performed pelvic examination and endocervical sampling using swab. Genital secretions were also obtained by self-collection using veil. Both clinician- and self-collected specimens were tested for HR-HPV DNA using Anyplex ${ }^{\text {TM }}$ II HPV28 genotyping test (Seegene, Seoul, South Korea). Acceptability and accuracy of both collection methods were assessed.

Results 253 women (mean, 35.0 years) were prospectively enrolled. HPV prevalence was $22.9 \%$, including $68.9 \%$ of HR-HPV (total HR-HPV prevalence: 15.8\%), with unusual HR-HPV genotypes distribution and preponderance (70\%) of HR-HPV targeted by Gardasil- $9^{\circledast}$ vaccine. Veil-based genital self-collection showed high acceptability (96\%), feasibility and satisfaction. Self-collection by veil was non-inferior to 
clinician-based collection for HR-HPV DNA testing, with 'good' agreement between both methods, high sensitivity (95.0\%; 95\%CI: $88.3-100.0 \%)$ and specificity $(88.2 \%$; 95\% CI: 83.9-92.6\%). Remarkably, the rates of HPV DNA and HR-HPV DNA positivity were significantly higher (1.67- and 1.57- fold, respectively) when using veil-based collection than clinician-collection.

Conclusion These observations highlight the unsuspected high burden of cervical oncogenic HR-HPV infection in Chadian women. Self-collection of genital secretions using the V-Veil-Up Gyn Collection Device constitutes a simple, highly acceptable and powerful tool to collect genital secretions for further molecular testing and screening of oncogenic HR-HPV that could be easily implemented in the national cervical cancer prevention program in Chad.

Disclosure No significant relationships.

\section{P825 HPV SEROPREVALENCE AND SEROCONVERSION AMONG HIV-POSITIVE MEN: COHORT STUDY IN SOUTH AFRICA}

${ }^{1}$ Admire Chikandiwa*, ${ }^{2}$ Helena Faust, ${ }^{3}$ Philippe Mayaud, ${ }^{2}$ Joakim Dillner, ${ }^{1}$ Sinead DelanyMoretlwe. 'Wits RHI, University of the Witwatersrand, Johannesburg, South Africa; ${ }^{2}$ Karolinksa Institute, Stockholm, Sweden; ${ }^{3}$ LSHTM, UK

10.1136/sextrans-2019-sti.870

Background The HPV seropositivity following natural infection can provide data on cumulative exposure to HPV. Studies evaluating seropositivity prospectively among men living with HIV (MLHIV) are few. We aimed to determine HPV type specific seroprevalence and seroconversion among MLHIV following natural HPV infection.

Methods We enrolled 304 sexually-active MLHIV $\geq 18$ years from Johannesburg. We collected socio-behavioral data, blood (CD4+ counts, HIV-1 plasma viral load [PVL] and serology), and genital swabs (HPV genotyping with Roche Linear Array and HPV 16/18 Viral Load [VL]) at enrolment and 6-monthly follow-up visits for up to 18 months. At enrolment and 18 months later, type-specific serum antibodies to $15 \mathrm{HPV}$ types (HPV6/11/16/18/31/33/35/39/45/52/56/58/59/68/73) were measured using HPV pseudovirions. Logistic regression evaluated factors associated with HPV seroconversion.

Results At enrolment, median age was 38 (IQR: 22-59) years, $25 \%$ reported $\geq 1$ sexual partners in the past 3 months and $5 \%$ reported ever having sex with other men. Most participants (65\%) were on ART, with median CD4+ count 445 cells/ $\mu \mathrm{L}$ (IQR: 328-567). Serology results were available for $99 \%$ of the 304 and 257 men who completed enrolment and 18 months visits. Seroprevalence of any HPV type was $66 \%$. Seropositivity for any HPV types of the bivalent (HPV16/18), quadrivalent (HPV6/11/16/18) and nonavalent (HPV6/11/16/ $1831 / 33 / 45 / 52 / 58)$ vaccines were $19 \%, 37 \%$ and $60 \%$ respectively. Among 59 men with genital HPV-DNA but seronegative for the same type at enrolment, 12 (22\%) had type-specific seroconversion at month 18 . Among these men, the risk of type specific seroconversion was higher among men with detectable PVL $(\mathrm{aOR}=2.78$, 95\%CI: 1.12-6.77), and HPV 18 $\mathrm{VL} \geq 5.3 \log 10 / 106$ cells ( $\mathrm{aOR}=3.32$, 95\%CI: 1.42-7.74).
Conclusion MLHIV have high HPV seroprevalence rates implying that prevention of HPV infection is required. There is evidence of seroconversion in response to detectable DNA infection at baseline, which was associated with both high HIV and HPV 18 viral loads.

Disclosure No significant relationships.

\section{P826 HIGH PREVALENCE OF CERVICAL HR-HPV IN IMMIGRANT WOMEN ORIGINATING FROM SUB- SAHARAN AFRICA AND LIVING IN ORLÉANS, FRANCE}

${ }^{1}$ Ralph Sydney Mboumba Bouassa*, ${ }^{2}$ Camelia Gubavu, ${ }^{3}$ Mathieu Matta, ${ }^{3}$ Leman Robin, ${ }^{3}$ David Veyer, ${ }^{2}$ Anne Gravier, ${ }^{2}$ Laurent Hocqueloux, ${ }^{2}$ Thierry Prazuck, ${ }^{3}$ Hélène Péré, ${ }^{3}$ Laurent Bélec. ${ }^{1} E$ Ecole Doctorale d'Infectiologie Tropicale, Tropicale Infectiologie, Franceville, Gabon; ${ }^{2}$ Service des Maladies Infectieuses et Tropicales, Centre Hospitalier Régional d'Orléans, Orléans, France; ${ }^{3}$ Laboratoire de Virologie, Hôpital Européen Georges Pompidou and Faculté de Médecine Paris Descartes, Université Paris Descartes (Paris V), Sorbonne Paris Cité, Paris, France

\subsection{6/sextrans-2019-sti.871}

Background We herein assess the HPV detection and HPVrelated cervical lesions in immigrant African women living in France.

Methods In 2018, immigrant African adult women attending for sexual health and HIV care a French healthcare facility, were included and subjected to endocervical swabs and Pap smear. HPV DNA was detected by multiplex real-time PCR (AnyplexTM II HPV28, Seegene, Seoul, South Korea).

Results 50 African immigrant women (mean age, 41.7) residing in France for an average of 10.7 years (range 1-32), including $74.0 \%$ of HIV-positive and $26.0 \%$ of HIV-negative, were prospectively included. HPV prevalence was $68.0 \%$, with $56.0 \%$ of HR-HPV and $24.0 \%$ of multiple HPV. Unexpectedly, the non-vaccine HR-HPV-68 (20.0\%) was the predominant genotype, followed by the Gardasil- $9^{\oplus}$ vaccine HR-HPV-58 (14.0\%). HPV-16 and HPV-18 were respectively detected in $6.0 \%$ and $10.0 \%$. HIV-infected women were more infected with HR-HPV (62.2\%) and multiple HR-HPV (16.2\%) than HIV-negative women (38.4\% and 7.7\%, respectively), $(\mathrm{P}=0.19$ and $\mathrm{P}=0.66$, respectively). Most women (84.0\%) showed normal cytology, but $16.0 \%$ exhibited cervical abnormalities, including $6.0 \%$ of Low-grade Squamous Intraepithelial Lesions (LSIL) and Abnormal Squamous Cells with Unknown Significance (ASCUS), and 4.0\% of High-grade Squamous Intraepithelial Lesions (HSIL). HPV-16 and HPV-18 were detected in one woman with HSIL. Finally, $87.5 \%$ of the study women with cervical abnormalities were HIV-positive, while only one HIV-negative woman showing cervical abnormalities.

Conclusion These findings highlight the unsuspected high burden of cervical HR-HPV infections often associated with cervical abnormalities in the first-generation immigrant women originating from sub-Saharan Africa and residing in France. These observations also point out the atypical epidemiological profile of cervical HPV infection with a rare and non-vaccine high-risk genotype being the most detected HPV, raising thus a concern about the low predictive efficacy of the Gardasil- $9^{\text {क }}$ vaccine in the prevention of the main genotypes circulating in these specific population residing in France.

Disclosure No significant relationships. 